\title{
A WAFER-LEVEL VACUUM PACKAGING PROCESS BY RTP ALUMINUM-TO-NITRIDE BONDING
}

\author{
Mu Chiao and Liwei Lin \\ Berkeley Sensor and Actuator Center \\ Department of Mechanical Engineering \\ University of California at Berkeley \\ Berkeley, CA 94720-1740 E-mail: muchiao@me.berkeley.edu
}

\begin{abstract}
This paper presents a wafer-level MEMS vacuum packaging process by using RTP (Rapid Thermal Processing) aluminum-to-nitride bonding. The measured quality factor of a vacuum-packaged comb-resonator is $1800 \pm 200$ corresponding to a $200 \mathrm{mTorr}$ vacuum inside the packaged micro cavity. Both long-term and accelerated vacuum stability tests are conducted to characterize the durability of the packages. It is found that the vacuum quality has not degraded over a period of 9 weeks under regular environment and over a period of 24 hours under a harsh environment $\left(130^{\circ} \mathrm{C}, 2.7 \mathrm{~atm}\right.$ and $\left.100 \% \mathrm{RH}\right)$ in the autoclave test. In an effort to address the issue of post-packaging frequency tuning, a pulsed laser deposition method is developed by using either indium or aluminum as the deposition material for frequency tuning. A maximum of $18.1 \%$ reduction in frequency has been achieved and a first-order, linear model is found adequate to explain the frequency tuning results.
\end{abstract}

\section{INTRODUCTION}

Vacuum packaging is an enabling technology for MEMS resonators to ensure high precision performances. For example, MEMS resonators have the advantages over conventional microelectronics for narrow bandwidth (high quality factor) applications in wireless communications[1]. Since the dominant energy loss of mechanical resonators in the micro scale is the air damping effect[2], vacuum packaging is key to achieve high quality factor. For example, the quality factor of comb-shape resonator is around 20-40 as measured in air and can be as high as 50000 in a $10^{-7}$ Torr vacuum[2].

Various vacuum packaging processes have been reported and drawbacks for practical implementation can be identified in previous approaches. For example, extra lithography and processing steps are required in a wafer-level, silicon nitride vacuum encapsulation process[1]. In the approach of localized resistive heating and bonding process, difficulty in wafer-level packaging[3] is identified. Recently, waferlevel RTP(Rapid Thermal Processing) bonding process for MEMS packaging applications is proven to be effective and easy for implementation with proven long-term stability
$[4,5]$. This paper advanced the RTP bonding technology further by demonstrating the feasibility of MEMS vacuum packaging.

In another packaging related issue, post-packaging trimming by means of pulsed laser deposition (PLD) is addressed. The background of the work comes from process variations in making microstructures, such as local geometrical variations and local mechanical property variations. As a result, it is difficult to precisely control the dynamic characteristics of micro resonators and postfabrication tuning is required. Several researches in the area of MEMS resonator tuning have been reported on two resonator components: spring and mass. In the spring tuning mechanisms, either thermal joule heating $[6,7]$ or electrostatic force pulling[8] have been proposed. In the mass tuning mechanisms, the MEMS resonator mass can be modified by localized CVD deposition[9]. A new approach for frequency tuning by using PLD(Pulsed Laser Deposition) is demonstrated in this paper. It has the advantages of precise process control, versatility and can be easily implemented into the packaging process as compared with previously demonstrated methods.

\section{VACUUM PACKAGING PROCESS AND RESULTS}

Figure 1(a) shows the schematic illustration of the RTP MEMS vacuum packaging process. Comb-shape microresonators are chosen as the vacuum packaging examples in this paper and a standard surface-micromachining process is used to fabricate these microresonators. One major design addition is that an integrated sealing ring using silicon nitride as the topmost layer in incorporated for the purpose of aluminum-to-nitride bonding[5]. The glass cap wafer is deposited and patterned with $4 \mu \mathrm{m}$-thick aluminum sealing rings. The aluminum ring width ranges from 100 to $250 \mu \mathrm{m}$ and bonding area ranges from 600x600 to 1000x1000 $\mu \mathrm{m}^{2}$. Before the vacuum packaging process, both the device and cap wafers are baked in vacuum at $300^{\circ} \mathrm{C}$ for various periods to dry out water and gas species adhere at the surface. Afterwards, the device and cap wafers are flip-chip assembled immediately and loaded into a quartz chamber as shown in Fig. 1(b). and put into a RTP chamber. The 


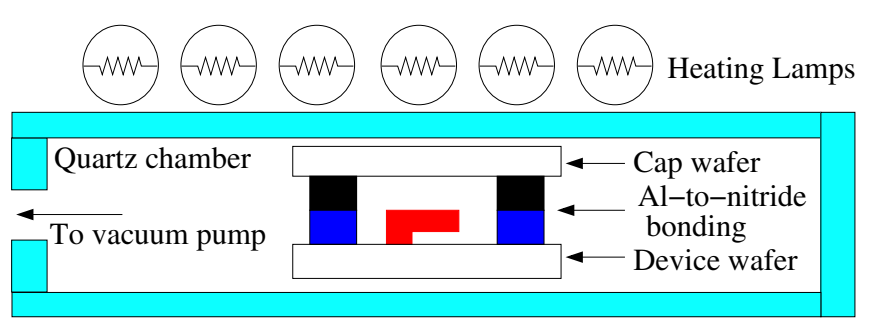

(a)

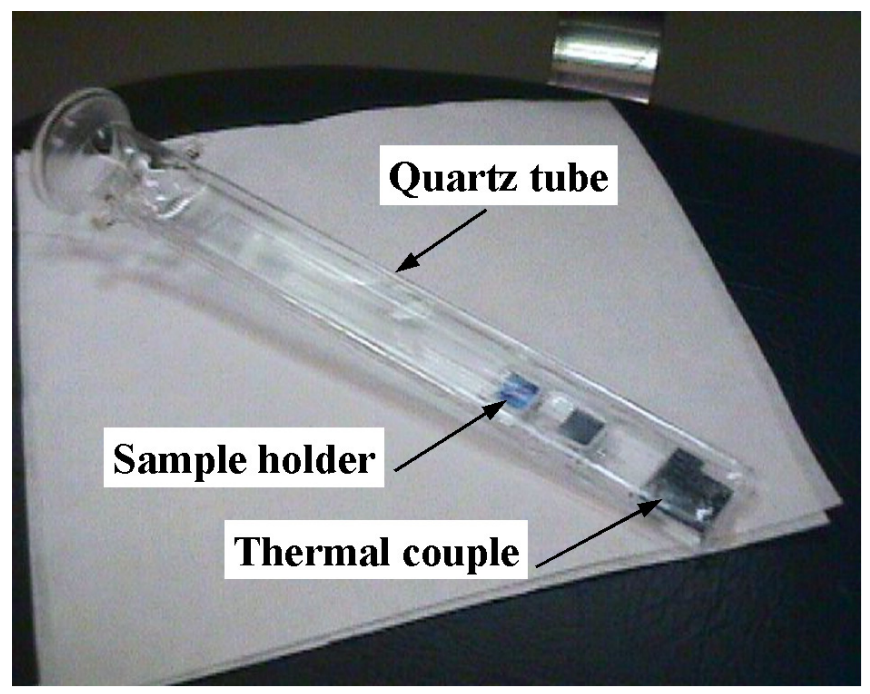

(b)

Figure 1: Experimental setup for RTP vacuum packaging. (a): The schematic diagram of the vacuum packaging process using RTP Al-to-nitride bonding. (b): Quartz chamber for RTP vacuum packaging.

base vacuum estimated at $10 \mathrm{mTorr}$ inside the quartz chamber is achieved by using a mechanical pump. After heating for 10 seconds at $750^{\circ} \mathrm{C}$, the aluminum-to-nitride bond is formed.

Figure 2 shows the measured spectrum of a vacuumpackaged, double-folded beam comb-drive resonator by using a micro-stroboscope[10]. The central resonant frequency is at about $18625 \mathrm{~Hz}$ and the quality factor is extracted as $1800 \pm 200$ corresponding to a pressure level about $200 \mathrm{~m}$ Torr inside the package[1]. In order to monitor the long-term stability of the vacuum packages, the quality factors of various devices are recorded up to 9 weeks, as shown in Fig. 3. Three vacuum-packaged micro resonators are tested. The quality factors of the resonators are 1800 , 400 and 200, corresponding to different pre-baking time in vacuum of 12, 4 and 0 hours, respectively. It can be observed in Fig. 3 that the quality factor increases when the pre-baking time period in vacuum increases. Furthermore, the vacuum levels hold up well under the regular operation environment because the value of the quality factor remains

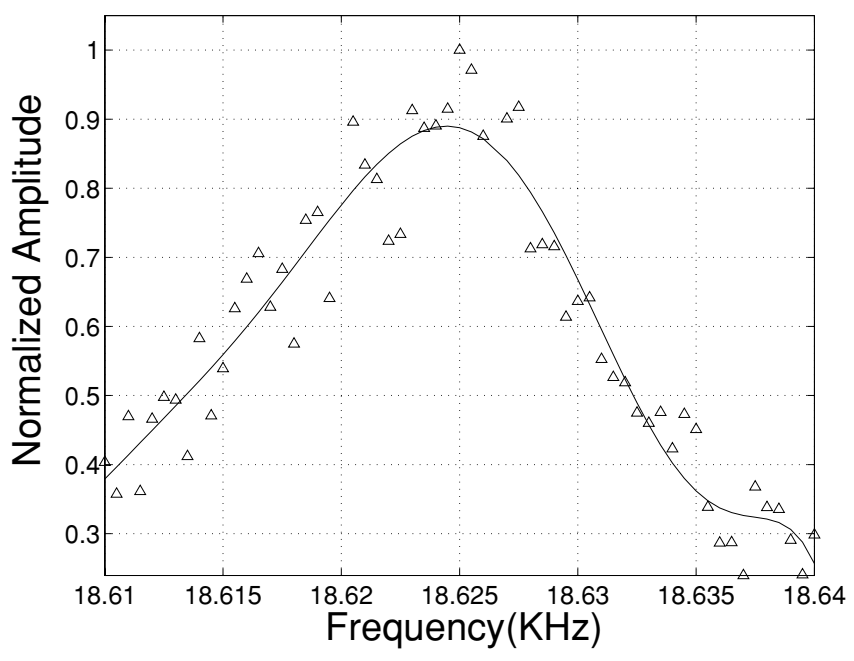

Figure 2: The spectrum of a vacuum packaged combresonator measured by a microstroboscope. The quality factor is extracted as $1800 \pm 200$ corresponding to a pressure of $200 \mathrm{mTorr}$ inside the package.

the same within experimental error bars. However, in order to determine an optimal pre-baking procedure, further investigations are necessary.

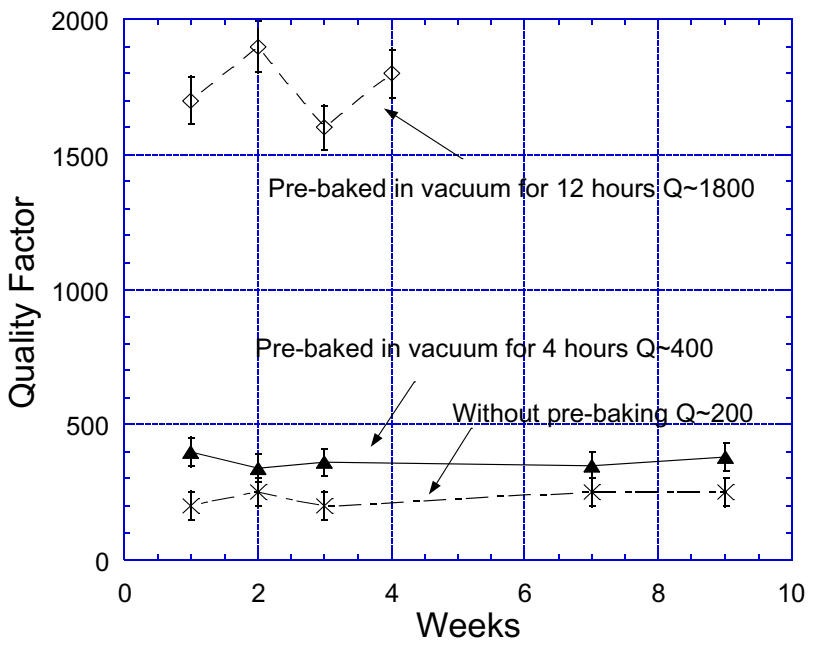

Figure 3: Long-term stability tests up to 9 weeks. Three sets of vacuum packaged resonators with different prebaking procedures are tested. The resulting $\mathrm{Q}$ inside the package increases with pre-baking time.

In addition to the regular operation environment, it is important to characterize the vacuum quality under harsh environment. Vacuum-packaged comb-resonators are put into harsh autoclave testing environment $\left(130^{\circ} \mathrm{C}, 2.7 \mathrm{~atm}\right.$ and $100 \% \mathrm{RH}$ ) for accelerated testing. The high pressure, high temperature and steam conditions could raise corrosion against the bonding interface and accelerate the gas diffusion process into the vacuum cavity. The spectrum of a comb resonator before and after a 24-hour continu- 
ously harsh environment testing are shown and compared in Fig. 4. In this case, the quality factor maintains at 200 after 24 hours of storage in the autoclave testing chamber. Since the slight differences between the two spectrum are within the normal experimental errors, a conclusion may be drawn that this harsh environment test does not affect the vacuum seal.

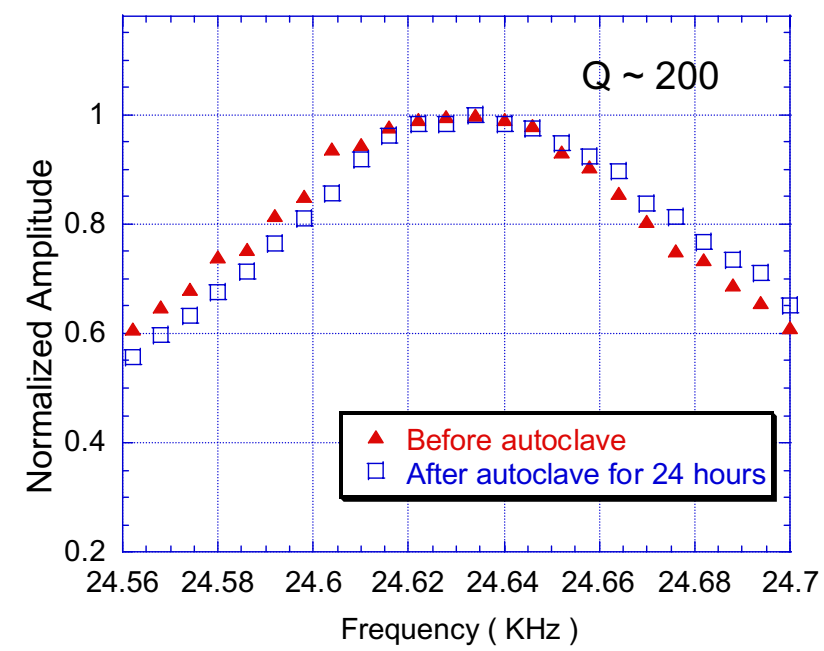

Figure 4: Accelerated testing results of a vacuum-packaged comb-resonator. The quality factor stays at 200 after 24 hours in the autoclave chamber under $130^{\circ} \mathrm{C}, 2.7 \mathrm{~atm}$ and $100 \%$ RH.

To further characterize the bonding quality, 60 MEMS packages formed by RTP Al-to-nitride bonding were sent to Sandia National Laboratory for MIL-STD-883E hermeticity testing[11]. The packaged devices are first tested using the gross leak check by using a combination of massincrease and bubble-check testing methods. Samples are immersed into 60 psi FC-72 fluid for 3 hours and weighted afterwards. An increase in weight will confirm a leakage into the packages. The samples are also heated over the FC-72 boiling temperature and the observation of bubble formation inside the packages will also confirm the leakage. After the packages have passed the gross leak tests, helium fine leak tests are performed. The packaged devices are first baked at $150^{\circ} \mathrm{C}$ for 2 hours to dry out water and put into a chamber with pressurized 60 psi helium for 2 hours. The packages are tested using a helium detector of a $10^{-8}$ atm-cc/sec scale. All 60 packages have passed the MIL-STD-883E gross and fine leak tests. The helium fine leak rate was found less than $5 \times 10^{-8} \mathrm{~atm}-\mathrm{cc} / \mathrm{sec}$. The examination on the quality of the bonding interface is conducted under SEM. Figure 5 shows the SEM photo of the silicon substrate where the bonding interface is examined after forcefully breaking the aluminum-to-nitride bond. It is observed that the glass debris is attached to substrate and fractures are formed in the silicon bulk. Part of the glass wafer is also fractured and attached to the silicon substrate as a strong bond is accomplished.

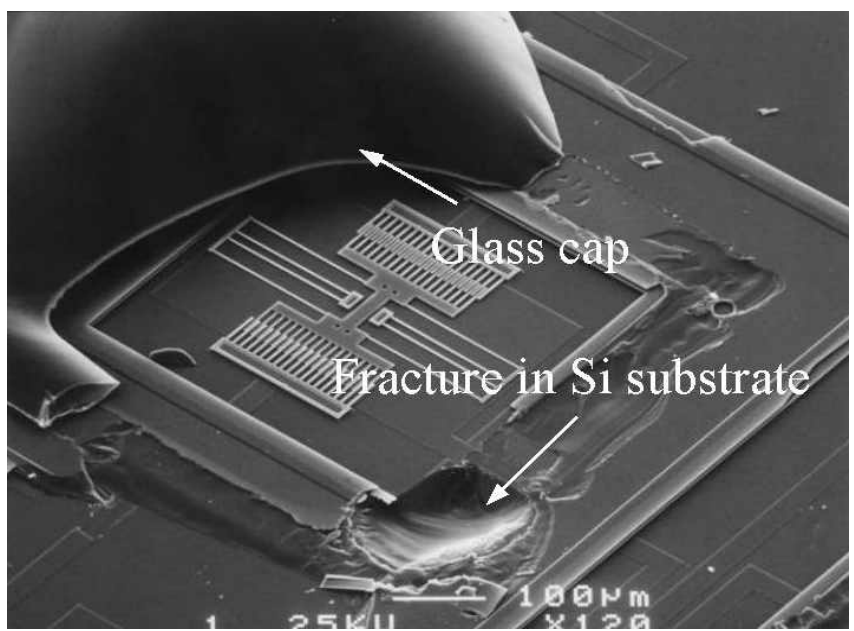

Figure 5: SEM microphoto of the silicon substrate after the Al-to-silicon nitride bond is forcefully broken. Bulk glass is found on the silicon substrate.

\section{POST-PACKAGING FREQUENCY TUNING BY PLD}

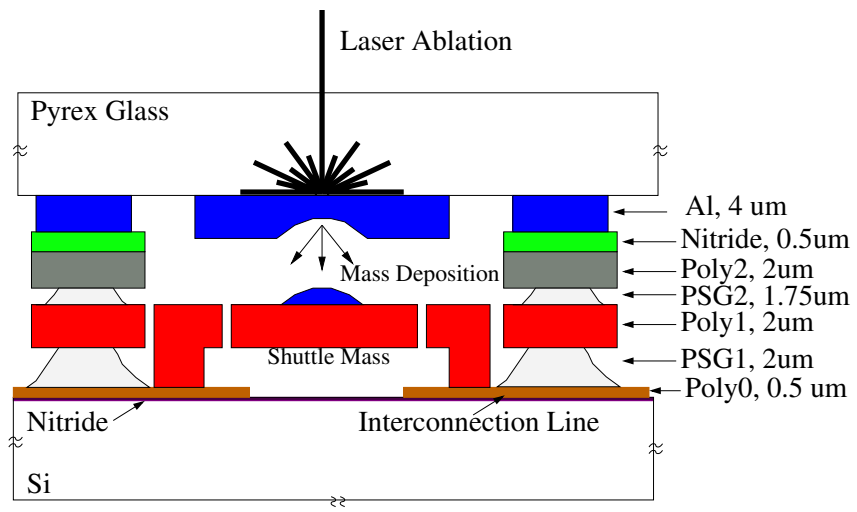

Figure 6: Schematic diagram showing the frequency tuning process by post-packaging PLD.

The working principle of the PLD frequency tuning process is illustrated in Fig. 6. After the MEMS packaging process is completed by using RTP Al-to-nitride bonding, a pulsed laser (6 nano-second, $532 \mathrm{~nm}, 0.6 \mathrm{~mJ})$ is introduced to locally vaporize the metal thin film that was deposited on the Pyrex-glass packaging cap. The same bonding ring material such as aluminum can be used as the PLD deposition material. A shuttle plate is designed as part of the resonator to allow the re-deposition of the donor metal such that a reduction in resonant frequency is expected. Under a high intensity and short pulse laser irradiation, the metal vapor can be built up at the metal film-glass interface $[12,13]$. Before the metal film layer is melted through-out, the vapor pressure is high enough to push and separate the rest of the metal film for deposition on to the accepting substrate as in the illustration. 
Both indium and aluminum have been used as the frequency tuning donor metal materials in this work. Figure.7 shows the SEM micrograph of a resonator after the PLD process by using indium as the deposition material. A chunk of indium is deposited on to a shuttle plate of a modified comb-shape resonator that has one side of the foldedbeam structure replaced with a shuttle plate. Figure 8 shows the measured spectrum of a resonator before and after the PLD tuning process and a $-1.2 \%$ of frequency change is observed. The quality factor after the PLD process seems to increase as shown in this figure and further investigation is required to understand the fundamental mechanism. It can be shown from a linear spring-mass vibration model that frequency change, $\Delta f, m_{o}$ and $m$ has the following relation,

$$
\Delta f=\frac{f-f_{o}}{f_{o}}=\sqrt{\frac{m_{o}}{m_{o}+\Delta m}}-1
$$

where $f_{o}$ and $f$ are the natural frequency of the microresonator before and after PLD, respectively. In this work, $m_{o}$, the original mass, is calculated using comb-resonator layout and $\Delta m$, the mass increment, is estimated by using an image processing software and SEM microphotos to estimate the deposition area and thickness. As shown in Fig. 9, a linear correspondence is observed as predicted by a the linear undamped spring-mass model(dashed line). A maximum frequency change of $-18.1 \%$ is achieved.

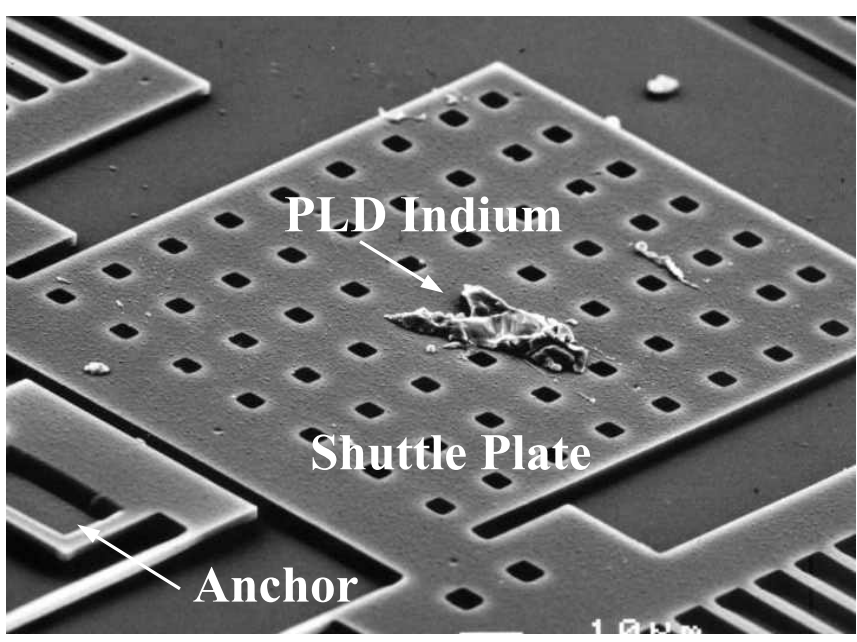

Figure 7: SEM microphoto of a MEMS resonator with mass deposited by PLD indium.

\section{CONCLUSIONS}

In this paper, MEMS vacuum packaging process based on RTP aluminum-to-silicon nitride bonding has been described. The quality factor of a vacuum-packaged combresonator has been measured as $1800 \pm 200$ corresponding to a pressure about $200 \mathrm{~m}$ Torr inside the package. The effect of pre-baking period in vacuum prior to bonding has been

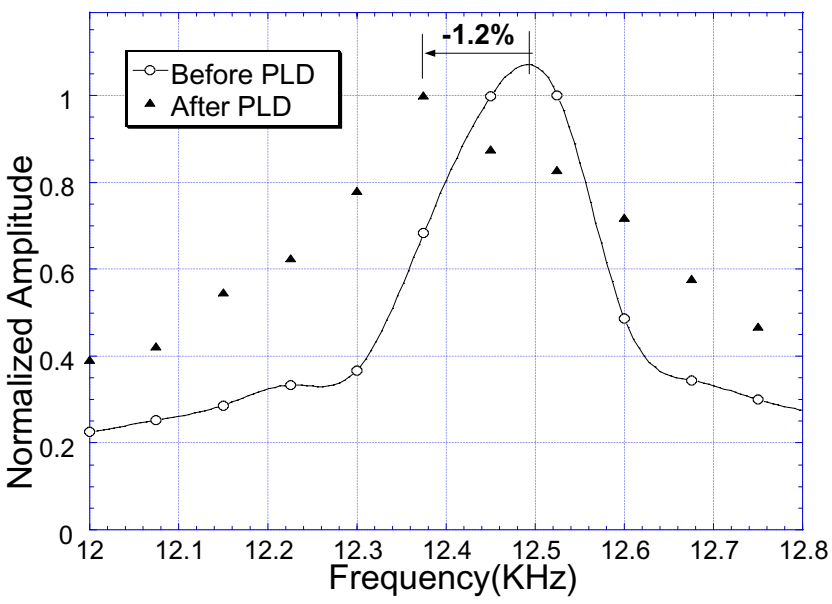

Figure 8: Spectrum measured by a microstroboscope of a resonator before and after PLD frequency tuning process.

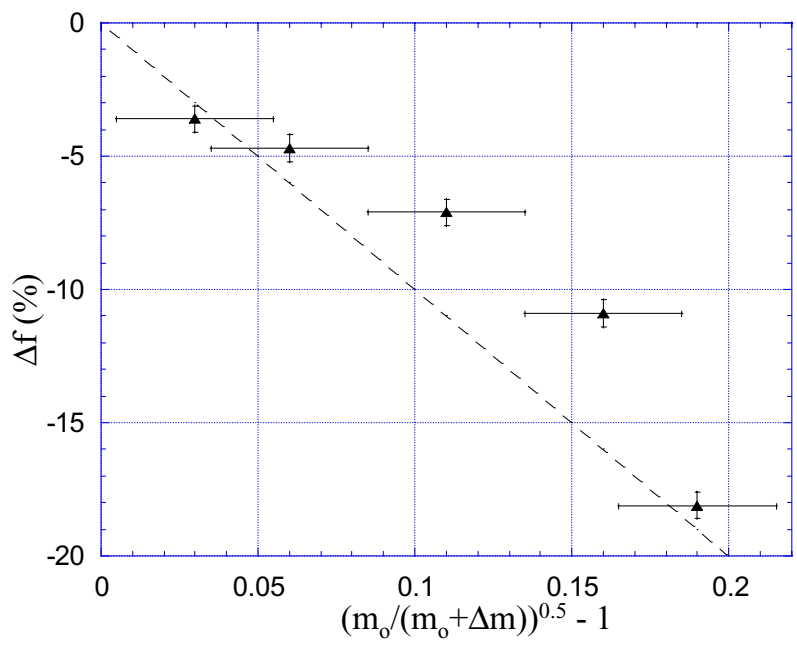

Figure 9: Experimental results showing a linear correspondence of frequency $\operatorname{shift}(\Delta \mathrm{f}$, in \%) with respect to $\left(m_{o} /\left(m_{o}+\Delta m\right)\right)^{0.5}-1$ as predicted by an undamped spring-mass model.

shown to have great effects on the vacuum encapsulation level. It is found that the quality factor increases with increasing pre-baking time under vacuum environment. The long-term stability tests of the vacuum packages have been carried out for up to 9 weeks. The $\mathrm{Q}$ remains stable over time and it indicates the vacuum quality has not degraded. Furthermore, vacuum-packaged comb-resonators have been placed in an autoclave chamber $\left(130^{\circ} \mathrm{C}, 2.7 \mathrm{~atm}\right.$ and $100 \% \mathrm{RH}$ ) for 24 hours for accelerated testing. It is found that the quality factor stays at 200 and the the vacuum quality inside the package has not been affected by the harsh environment.

A post-packaging frequency tuning technique by PLD has been demonstrated by using either indium and or aluminum as the donor material. The spectrum of a MEMS 
resonator is measured before and after the PLD frequency tuning process and a maximum resonant frequency reduction of $18.1 \%$ on a modified comb-shape resonator is demonstrated. It is found that a first-order, undamped spring-mass vibration model explains well for the PLD tuning experimental results.

\section{ACKNOWLEDGEMENTS}

The authors would like to thank Dr. A. D. Oliver of Sandia National Laboratory for MIL-STD-883E hermeticity testing and discussions on leak tests, Mr. S. Avadhanula and Prof. R. Fearing of EECS, UC-Berkeley on their laser equipment for the PLD work and Dr. X. Meng of Cryogenic group at UCB for the vacuum apparatus. Theses devices are fabricated at the UCB Microfabrication Laboratory. This work is supported in part by an NSF CAREER award(ECS-0096098) and a DARPA MTO/MEMS grant (F30602-98-2-0227).

\section{REFERENCES}

[1] L. Lin, R. T. Howe, and A. P. Pisano. Microelectromechanical filters for signal processing. Journal of Microelectromechanical Systems, 7(3):286-294, Sept. 1998.

[2] W. C. Tang. Electrostatic Comb Drive for Resonant Sensor and Actuator Applications. PhD thesis, University of California at Berkeley, 1990.

[3] Y.T. Cheng, W. T. Hsu, L Lin, C. T. Nguyen, and K Najafi. Vacuum packaging technology using localized aluminum/silicon-to-glass bonding. In Proceedings of IEEE Micro Electro Mechanical Systems, pages 18-21, Jan 2001.

[4] M. Chiao and L. Lin. Hermetic wafer bonding based on rapid thermal processing. Sensors and Actuators A-Physical, 91:398-402, 2001.

[5] M. Chiao and L. Lin. Accerlerated hermeticity testing of a glass-silicon package formed by RTP aluminumto-silicon nitride bonding. In 11th Int. Conference on Solid-State Sensors and Actuators, Transducer's 01 , Technical Digest, pages 190-193, Munich, Germany, 2001.

[6] K. Wang, A. C. Wong, W. T. Hsu, and C. T.-C. Nguyen. Frequency trimming and Q-factor enhancement of micromechanical resonators via localized filament annealing. In Transducers'97, pages 109-112, 1997.

[7] T. Remtema and L. Lin. Active frequency tuning for microresonators by localized thermal stressing effects. In Technical Digest of Solid-State sensor and actuator workshop, pages 363-366, 2000.

[8] K. B. Lee and Y. H. Cho. Frequency tuning of a laterally driven microresonator using an electrostatic comb array of linearly varied length. In Transducers'97, pages 113-116, 1997.

[9] D. Joachim and L. Lin. Localized deposition of polysilicon for MEMS post-fabrication processing. In Proceedings of ASME International Mechanical Engineering Congress and Exposition-MEMS, pages 37-42, 1999.

[10] D. M. Freeman and C. Q. Davis. Using video microscopy to characterize micromechanics of biological and man-made micromachines. In Technical Digest of Solid-State sensor and actuator workshop, pages 161167, June 1996.

[11] A.D. Oliver and C.M. Matzke. 100\% foundry compatible packaging and full wafer release/die seperation technique for surface micromachined devices. In Late News Digest of Solid-State sensor and actuator $w$ orkshop, pages 5-6, 2000.

[12] R. J. Harrach. Analytical solutions for laser heating and burnthrough of opaque solid slabs. Journal of Applied Physics, 48(6):2370-2383, June 1977.

[13] J. Bohandy, B. F. Kim, F. J. Adrian, and A. N. Jette. Metal deposition st $532 \mathrm{~nm}$ using a laser transfer technique. Journal of Applied Physics, 63(4):1158-1162, Feb. 1988 . 